\title{
COMUNICAÇÃO
}

\section{COMPARAÇÃO ENTRE DOCES PRODUZIDOS A PARTIR DE FRUTOS DE DIFERENTES ESPÉCIES E CULTIVARES DE MARMELEIRO (Cydonia oblonga MILLER E Chaenomeles sinensis KOEHNE) ${ }^{1}$}

\author{
Comparation among marmalades produced from different fruit quince species \\ (Cydonia oblonga Miller and Chaenomeles sinensis Koehne) and cultivars \\ Ângelo Albérico Alvarenga ${ }^{2}$, Enilson Abrahão ${ }^{3}$, Rafael Pio $^{4}$, Francinely Aparecida Assis $^{5}$, \\ Nilton Caetano de Oliveira ${ }^{6}$
}

\begin{abstract}
RESUMO
Objetivou-se com este trabalho comparar doces (marmeladas) produzidos a partir de frutos de diferentes cultivares de marmeleiro, como também os possíveis ganhos obtidos com a mistura de polpa dos frutos das cultivares. Os frutos foram colhidos em uma coleção de cultivares de marmeleiros pertencente à EPAMIG, localizada no município de Maria da Fé (MG), nos meses de janeiro a abril de 2005. Os frutos foram processados artesanalmente dando origem às marmeladas. Os tratamentos foram constituídos pelas marmeladas das cultivares Mendoza, Japonês, Provence e pela mistura das polpas das cultivares Portugal, Provence e Mendoza com a Japonês, comparadas com a marmelada tradicional feita a partir da 'Portugal'. Foi feita uma avaliação sensorial das características cor, brilho, sabor, textura e aroma das marmeladas por um grupo de vinte pessoas. A análise estatística dos dados, com o respectivo teste de médias, mostrou uma superioridade da marmelada da cultivar Mendoza sobre as demais com relação ao brilho. Quanto aos itens sabor e textura, a marmelada da 'Mendoza' foi superior apenas a da 'Japonês', sendo que as misturas da 'Japonês' com as cultivares Mendoza e Provence se igualaram à da 'Mendoza'. Com relação ao aroma não foram detectadas diferenças entre as cultivares. Concluiu-se assim que a 'Mendoza' proporcionou a obtenção da melhor marmelada e que a mistura com outras polpas melhorou as qualidades da marmelada da cultivar Japonês.
\end{abstract}

Termos para indexação: Marmelo, marmelada, fruta, doce, análise sensorial, qualidade.

\begin{abstract}
The present study was made to compare marmalades produced from different quince fruit cultivars, as well as, the earnings possible from different pulp mixtures. Fruits were collected on EPAMIG's quince collection yard, at district of Maria da Fé, MG, in from January to April, 2005. Marmalades were manually processed. Treatments were marmalades from Mendoza, Japonês, Provence cultivars and by pulp mixture of Portugal, Provence and Mendoza plus the Japonês quince cultivars, compared to traditional marmalade from Portugal cultivar. Sensorial characteristic evaluation as color, bright, taste, texture and flavor were made for twenty people. Data statistic analyses and average tests showed superior bright quality of the marmalade Mendoza cultivar in relation all others. Mendoza marmalade flavor and texture were superior only to Japonês marmalade; however, there were no difference in marmalade qualities when a mixture of Japonês and others pulp cultivars was made. There were found no flavor differences among the cultivars. So, the Mendoza cultivar produced the best marmalade and mixture of other quince pulps improved the Japonês marmalade.
\end{abstract}

Index terms: Quince, marmalade, fruit, candy, sensory test, quality.

(Recebido em 31 de janeiro de 2006 e aprovado em 6 de outubro de 2006)

Dentre as fruteiras de clima temperado, o marmelo é, sem dúvida, uma das mais preciosas e apreciadas frutas em todo o mundo, principalmente pela larga aplicação na industrialização, na fabricação de marmeladas, compotas e geléias (RIGITANO, 1957).

\footnotetext{
${ }^{1}$ Apoio financeiro: FAPEMIG e CNPq

2Doutor, Pesquisador - EPAMIG/CTSM - Cx. P. 176 - 37200-000 - Lavras, MG - angelo@epamig.br

${ }^{3}$ Mestre, Pesquisador - EPAMIG/CTSM - Cx. P. 176 - 37200-000 - Lavras, MG - enilson@epamig.br

${ }^{4}$ Doutor - Centro de Ciências Agrárias - Universidade Estadual do Oeste do Paraná/UNIOESTE - Cx. P. 1008 - 85960-000 - Marechal Cândido Rondon, PR rafaelpio@hotmail.com

${ }^{5}$ Graduanda em Agronomia - Rua Rodolfo Blummer, 44, Jardim Glória - 37200-000 - Lavras, MG - franscinel@agronomia.ufla.br - Bolsista BIC/ FAPEMIG

${ }^{6}$ Graduado - EPAMIG - Fazenda Experimental de Maria da Fé, Bairro Vargedo - 35517-000 - Maria da Fé, MG - niltoncaetano@epamig.br
} 
Introduzidos no Brasil, em 1532, por Martim Afonso de Souza, os marmelos e a marmelada foram os principais e os primeiros produtos de exportação paulista, antecessores ao café, sendo os doces comercializados em caixas e caixetas. No mundo antigo e no Brasil colonial, poucos frutos como os do marmeleiro tiveram tão relevante papel, entretanto, atualmente é difícil encontrar uma frutífera com seu valor histórico-social tão pouco difundida e estudada. As causas prováveis desse pequeno interesse devem residir na utilização pouco nobre do marmelo, máximo como matéria-prima industrial e no incipiente consumo natural como já relatado por Rigitano (1957).

Em Minas Gerais, a marmelocultura teve seu apogeu no Sul do Estado na década de 30, quando havia na região mais de 7.000 hectares cultivados com 27 agroindústrias somente para o processamento exclusivo de marmelos, sendo os principais municípios produtores Marmelópolis, Delfim Moreira, Virgínia, Cristina e Maria da Fé. Entretanto, a cultura do marmeleiro foi quase que totalmente dizimada por uma doença conhecida como "Requeima" ou "Entomosporiose", além da falta de incentivos aos produtores de frutas e queda dos preços pagos pelas indústrias (ABRAHÃO et al., 1996b). Atualmente o Estado possui apenas 160 hectares cultivados com marmeleiros, apesar de ainda ser o maior produtor nacional (AGRIANUAL, 2004).

Por causa da baixa produção nacional, a quantidade de frutas que chega ao mercado pode ser comercializada a preços atraentes. Além disso, é uma alternativa na diversificação de propriedades rurais, podendo agregarse valor ao produto na elaboração de doces, principalmente quando empregado em cooperativas regionais de produtores e comercializados em mercado local.

O destaque para os frutos de marmelo é a alta quantidade de pectina, o que vem a facilitar a confecção de marmeladas e geléias. O consumo de marmelos é aconselhável para o bom funcionamento do aparelho digestivo, sendo altamente recomendável na recuperação de pessoas debilitadas. Estudos atuais têm confirmado essas propriedades da marmelada. Ferreira et al. (2004) em Portugal, compararam 18 tipos comerciais de marmeladas quanto à sanidade microbiológica, características físicoquímicas e avaliação sensorial. A análise sensorial mostrou que as amostras se classificavam em duas classes, uma com aroma e gosto típico, atribuído a textura com grânulos pequenos com superfície brilhante em contraste à outra com aroma intenso, porém menos típico, mais ácida e com textura mais granulosa.

Linkevich \& Markh (2005) mostraram que o suco e produtos obtidos do marmelo apresentam propriedades antibióticas, inibindo o desenvolvimento de esporos de bactérias, inclusive da Salmonela.

Silva et al. (2004a), também pesquisando frutos de marmelo, constataram a existência de diversos compostos fenólicos, ácidos orgânicos e aminoácidos livre que, mesmo após a transformação em marmeladas ainda permanecem em grande quantidade. Em trabalhos mais recentes, Silva et al. (2004b) mostraram que estes componentes da marmelada apresentam importante atividade antioxidante, altamente benéficos à saúde humana.

Alvarenga et al. (1994) analisaram a constituição físico-química dos frutos maduros das cultivares Japonês e Portugal. O peso do fruto variou de 211 a $88 \mathrm{~g}$, o teor de umidade de 81 a $90 \%$, o amido de 2,68 a $11 \%$, os açúcares totais de 8,53 a 2,98\%, a acidez titulável de 1,44 a $0,30 \%$, os fenólicos totais de 1,172 a $0,0165 \%$ e a pectina total de 460 a $650 \mathrm{mg} / 100 \mathrm{~g}$, nas cultivares Japonês e Portugal, respectivamente. Os valores encontrados ressaltam a grande variação entre os frutos dos marmeleiros do gênero Chaenomeles e Cydonia, com consequiência direta na qualidade da marmelada.

Abrahão et al. (1996a) compararam a tradicional marmelada nacional produzida a partir de marmelos da cultivar Portugal com a da 'Japonês', bem como uma obtida com a mistura dessas duas cultivares. Foram feitas avaliações sensoriais do sabor, textura, visual, ponto e geral. Eles concluíram que doces produzidos com base na mistura dessas duas cultivares são bastante semelhantes ao da tradicional com as mesmas qualidades.

O sabor é determinado pela combinação de quatro sensações básicas: doce, ácido, salgado e amargo. A doçura está diretamente ligada aos açúcares e à relação açúcares/acidez. O sabor azedo é determinado pela presença de ácidos orgânicos e o amargo determinado pela presença de adstringência, resultado dos taninos e compostos fenólicos. Por sua vez, a cor é um efeito visual decorrentes dos diferentes comprimentos de ondas refletidos pelo produto (PINHEIRO, 2003). O aroma, por sua vez é outro importante atributo dos alimentos, derivado da volatilização de seus constituintes.

O presente trabalho pretende comparar doces (marmelada) produzidos com base em frutos de diferentes variedades de marmelo, bem como das misturas de polpas dessas cultivares.

Com esse objetivo, frutos das cultivares de marmelo Portugal, Provence, Mendoza (INTA 37) e Japonês foram colhidos de plantas existentes em uma coleção de variedades na Fazenda Experimental de Maria da Fé, pertencente a EPAMIG, no período referente à safra 2004/ 2005. Os frutos das variedades Portugal, Provence e 
Mendoza foram colhidos em janeiro, enquanto os da 'Japonês', de produção tardia (ABRAHÃO et al., 1995), colhidos em abril de 2005.

Uma vez colhidos, os frutos foram partidos ao meio para a retirada das sementes. Para a confecção das marmeladas foi utilizado o método de fabricação caseira. As polpas foram colocadas em tacho de cobre com água, o suficiente para cobri-las, e a seguir colocado ao fogo para a obtenção da massa. Após a obtenção da massa, esperou-se o seu esfriamento e a seguir peneirou-se a mesma em peneira com malha de $1 \mathrm{~mm}$. Em seguida, fez-se a pesagem da massa, retornando-a ao fogo, acrescida de 650 gramas de açúcar comum, para cada quilograma de massa. O tacho foi retirado do fogo quando a massa começou a se desgarrar do mesmo ao se passar a colher de pau, utilizada para mexer durante todo o processo de cozimento. Logo em seguida, a massa foi colocada em caixas de madeira, protegidas com plástico. Esperou-se esfriar e finalmente o produto foi embalado em plástico transparente.

Foram feitos sete tipos de marmeladas, sendo um referente a cada variedade e mais três, oriundas das misturas de massas das variedades 'Portugal', 'Provence' e 'Mendoza' com a 'Japonês' na proporção de 50\%. Essa mistura foi realizada com o objetivo de verificar o possível ganho a ser obtido pela marmelada da cultivar Japonês com a adição de polpa de outras cultivares.

Foram retiradas 3 amostras de cada uma das cultivares e enviadas a 3 laboratórios para análise. Foram analisados os sólidos solúveis, a acidez, o pH, os açúcares solúveis, o teor de fibras e os resíduos minerais. Teste de cor dos produtos também foi realizado.

Para a determinação da cor foi utilizado um colorímetro marca Minolta, modelo CR 300, com a determinação dos valores $\mathbf{a}$ e $\mathbf{b}$, onde $\mathbf{a}$ está relacionado à intensidade de verde $(-80)$ a vermelho $(+100)$ e b com a intensidade de azul (-70) a amarelo (+70). $\mathrm{O}$ valor de $\mathbf{L}$ por sua vez indica a luminosidade, diferenciando cores claras e escuras, variando de "zero" para o negro a 100 para o branco.

A análise sensorial foi realizada por provadores não treinados. Foi usado um teste de comparação múltipla (MORAES, 1985), que teve como padrão a marmelada da 'Portugal' comparada as demais cultivares e misturas. Participaram do teste 20 pessoas que avaliaram os diversos tratamentos quanto às características de cor, brilho, sabor, textura e aroma. Para cada uma dessas características os avaliadores atribuiram notas de 1 a 5 conforme escala abaixo:

1 - Qualidade da amostra inferior ao padrão;
2 - Qualidade da amostra ligeiramente inferior ao padrão;

3 - Qualidade da amostra igual ao padrão;

4 - Qualidade da amostra ligeiramente superior ao padrão;

5 - Qualidade da amostra superior ao padrão.

Cada provador constituiu uma repetição dentro do delineamento estatístico inteiramente ao acaso. Os dados obtidos foram submetidos à análise estatística e as médias comparadas pelo teste de Tukey ao nível de 5\% de significância.

Pela Tabela 1, verificam-se os resultados das análises físico-químicas realizadas nas marmeladas das diferentes cultivares e misturas. Esses resultados servem de auxílio para a compreensão dos demais resultados obtidos na análise sensorial.

\section{Quanto à cor:}

A análise estatística dos resultados detectou diferenças entre as marmeladas produzidas a partir de frutos das diferentes cultivares e a da cultivar padrão ('Portugal') (Tabela 2). Dentre as características analisadas, a cor teve uma maior diferenciação dos tratamentos. A marmelada da cultivar Mendoza alcançou a pontuação $(3,95)$, porém não diferiu da cultivar Japonês. Através das avaliações feitas no colorímetro (Tabela 1), pode-se verificar que as marmeladas feitas a partir da cultivar Japonês $(\mathrm{L}=35,56)$ e suas misturas ficaram mais claras em relação às outras cultivares e ao padrão $(\mathrm{L}=25,10)$. Essa diferença na cor, por sua vez, pode ser oriunda da diferença na constituição dos frutos dos marmeleiros do gênero Cydonia e Chaenomeles, conforme mostrada por Alvarenga et al. (1994).

\section{Quanto ao brilho:}

Com relação ao brilho das marmeladas (Tabela 2), observou-se um maior valor para a cultivar Mendoza em relação ao apresentado pelas demais, assim como, de suas misturas com a 'Japonês'. Os frutos do marmeleiro 'Japonês' conferiram uma característica mais opaca à marmelada, mesmo quando misturados aos de outras cultivares. A 'Mendoza'foi a cultivar que mais se assemelhou à comercialmente produzida ('Portugal').

\section{Quanto ao sabor:}

A cultivar Mendoza alcançou a maior pontuação, porém, diferiu significativamente apenas da 'Japonês' e da mistura da 'Japonês' x 'Portugal' (Tabela 2). A cultivar Provence não se diferenciou da 'Mendoza', mas foi superior à 'Japonês'. Alvarenga et al. (1994), analisando as 
Tabela 1 - Valores dos teores de sólidos solúveis, acidez, pH, açúcares solúveis, fibras, resíduo mineral e intensidade de cores (L, a e b) encontrados nas marmeladas provenientes das diferentes cultivares. EPAMIG/CTSM, Lavras-MG, 2005 .

\begin{tabular}{|c|c|c|c|c|c|c|c|c|c|}
\hline \multirow[b]{2}{*}{ Cultivares $^{(1)}$} & \multirow{2}{*}{$\begin{array}{l}\text { Sólidos } \\
\text { Solúveis } \\
\text { (Brix) }\end{array}$} & \multirow{2}{*}{$\begin{array}{c}\text { Acidez } \\
(\mathrm{meq} / 100 \mathrm{~g})\end{array}$} & \multirow[b]{2}{*}{$\mathrm{pH}$} & \multirow{2}{*}{$\begin{array}{c}\text { Açúcares } \\
\text { Solúveis } \\
(\%)\end{array}$} & \multirow{2}{*}{$\begin{array}{c}\text { Fibras } \\
(\%)\end{array}$} & \multirow{2}{*}{$\begin{array}{l}\text { Resíduo } \\
\text { Mineral } \\
(\%)\end{array}$} & \multicolumn{3}{|c|}{ Cor } \\
\hline & & & & & & & $\mathrm{L}$ & $\mathrm{a}$ & $\mathrm{b}$ \\
\hline Portugal $^{(2)}$ & 73,77 & 11,78 & 3,5 & 55,30 & 4,27 & 0,220 & 25,10 & 12,43 & 0,38 \\
\hline Mendoza & 81,18 & 7,63 & 3,8 & 60,79 & 6,40 & 0,437 & 25,57 & 11,30 & 2,58 \\
\hline Japonês & 75,56 & 10,61 & 3,6 & 58,05 & 4,67 & 0,273 & 35,56 & 10,21 & 1,16 \\
\hline Provence & 74,61 & 9,01 & 3,7 & 54,47 & 5,03 & 0,207 & 27,12 & 11,09 & 0,70 \\
\hline $\begin{array}{l}\text { Mendoza X } \\
\text { Japonês }\end{array}$ & 73,93 & 11,27 & 3,7 & 62,83 & 5,50 & 0,353 & 31,63 & 9,60 & 0,93 \\
\hline $\begin{array}{l}\text { Provence X } \\
\text { Japonês }^{(3)}\end{array}$ & 73,79 & 13,36 & 3,6 & 56,89 & 5,53 & 0,300 & 33,75 & 5,99 & 2,63 \\
\hline $\begin{array}{l}\text { Portugal X } \\
\text { Japonês }^{(3)}\end{array}$ & 72,82 & 10,10 & 3,7 & 61,56 & 4,13 & 0,237 & 30,95 & 10,39 & 1,69 \\
\hline
\end{tabular}

${ }^{(1)}$ Os valores apresentados são referentes a média da análise de 3 amostras.

${ }^{(2)}$ Cultivar considerada como Padrão.

${ }^{(3)}$ Marmelada produzida com a mistura de polpa dos frutos das duas cultivares em igual proporção.

Tabela 2 - Avaliação sensorial comparativa de marmeladas produzidas a partir de frutos de diferentes cultivares de marmeleiro com a da 'Portugal', considerada como padrão. EPAMIG/CTSM, Lavras-MG, 2005.

\begin{tabular}{llllll}
\hline \multirow{2}{*}{ Tratamento } & \multicolumn{5}{c}{ Características } \\
\cline { 2 - 6 } & \multicolumn{1}{c}{ Cor $^{(2)}$} & Brilho & Sabor & Textura & Aroma \\
\hline 'Mendoza' & $3,95 \mathrm{a}^{(3)}$ & $3,85 \mathrm{a}$ & $3,40 \mathrm{a}$ & $3,60 \mathrm{a}$ & $3,10 \mathrm{a}$ \\
'Japonês' & $3,30 \mathrm{ab}$ & $1,90 \mathrm{~b}$ & $1,75 \mathrm{c}$ & $1,75 \mathrm{c}$ & $2,55 \mathrm{a}$ \\
'Provence' & $2,90 \mathrm{bc}$ & $2,20 \mathrm{~b}$ & $2,75 \mathrm{ab}$ & $2,40 \mathrm{bc}$ & $2,60 \mathrm{a}$ \\
'Mendoza' x 'Japonês',(1) & $2,50 \mathrm{bcd}$ & $2,00 \mathrm{~b}$ & $2,60 \mathrm{abc}$ & $2,85 \mathrm{ab}$ & $2,70 \mathrm{a}$ \\
'Provence' x 'Japonês' ${ }^{(1)}$ & $1,95 \mathrm{~cd}$ & $1,85 \mathrm{~b}$ & $2,65 \mathrm{abc}$ & $2,80 \mathrm{abc}$ & $2,85 \mathrm{a}$ \\
'Portugal' x 'Japonês' ${ }^{(1)}$ & $1,80 \mathrm{~d}$ & $1,65 \mathrm{~b}$ & $2,40 \mathrm{bc}$ & $2,50 \mathrm{bc}$ & $2,40 \mathrm{a}$ \\
\hline CV $(\%)^{(4)}$ & 38,58 & 41,23 & 39,18 & 48,83 & 34,39 \\
\hline DMS $^{(5)}$ & 0,97 & 0,85 & 0,93 & 1,07 & 0,85 \\
\hline
\end{tabular}

(1) Tratamento referente a mistura de polpa do fruto das cultivares em igual proporção.

(2) Teste comparativo de qualidade, onde 1 é inferior ao padrão; 2 é ligeiramente inferior ao padrão; 3 é igual ao padrão; 4 é ligeiramente superior ao padrão e 5 é superior ao padrão.

${ }^{(3)}$ Médias nas colunas, seguidas de letras diferentes, indicam diferenças significativas pelo teste de Tukey $(\mathrm{p}<0,05)$.

(4) Coeficiente de Variação

(5) Diferença Mínima Significativa das médias

composições físico-químicas dos frutos de marmeleiros, observaram na cultivar Japonês uma maior acidez e teor de fenólicos totais em relação à 'Portugal'. A análise das marmeladas (Tabela 1) também mostra que a 'Mendoza' apresentou uma baixa acidez associada a um alto teor de sólidos solúveis. Tais diferenças podem ter afetado o sabor da marmelada da cultivar Japonês, o que vem a corroborar os resultados aqui obtidos. Abrahão et al. (1996a), comparando marmeladas das cultivares Portugal e Japonês, relataram que $67 \%$ dos provadores caracterizaram o sabor 
da 'Portugal' como ótimo, enquanto para $41 \%$ dos mesmos provadores o sabor da 'Japonês' foi considerado muito bom.

\section{Quanto à textura:}

A cultivar Mendoza apresentou o maior valor médio, não se diferenciando das misturas 'Japonês' $x$ 'Mendoza' e 'Japonês' $x$ 'Provence'. As marmeladas da 'Provence' e das misturas 'Provence' $x$ 'Japonês' e 'Portugal' x 'Japonês' não tiveram valores significamente superiores à 'Japonês'. Em estudos preliminares, Abrahão et al. (1996a) relataram que a cultivar Japonês deu origem a uma marmelada com textura mais grosseira, considerada por muitos provadores como a sensação semelhante a grânulos de areia. No presente trabalho, procedeu-se uma peneiragem (malha fina $=1 \mathrm{~mm}$ ) das massas obtidas dos frutos de todos os tratamentos, antes da confecção das marmeladas. Assim, observa-se pelos resultados alcançados que, se a marmelada da 'Japonês' por um lado foi inferior a da 'Mendoza', por outro, apresentou as mesmas qualidades das demais cultivares, mostrando um efeito positivo da peneiragem em sua textura.

A adição de polpa das cultivares Mendoza e Provence à 'Japonês' produziu marmeladas com características de sabor e textura semelhantes à 'Mendoza', conforme pode ser observado na Tabela 2. Abrahão et al. (1996a) também comprovaram esse efeito por meio da mistura de polpa das cultivares Japonês e Portugal.

\section{Quanto ao aroma:}

Para esta característica, não se observaram diferenças entre as marmeladas nos diversos tratamentos aplicados. Todas as marmeladas tiveram aroma com valores semelhantes ao do padrão ('Portugal').

Pode-se concluir que, em termos de qualidade sensorial, a cultivar Mendoza pode substituir a tradicional 'Portugal' para a fabricação de marmelada.

Os autores agradecem ao Sr. Antônio Barbosa e a Dona Terezinha Botrel Barbosa pela colaboração na confecção artesanal das marmeladas, ao Professor Dr. Paulo Roberto Clemente da UFLA pelo apoio e sugestões, como também aos funcionários da EPAMIG e UFLA Renata, Sílvio, Samuel e Tina pelo auxílio nas análises laboratoriais.

\section{REFERÊNCIAS BIBLIOGRÁFICAS}

ABRAHÃO, E.; ALVARENGA, A. A.; SOUZA, M. de. Produção tardia de marmeleiro (Chaenomeles sinensis) cv. Japonês, na região Sul do Estado de Minas Gerais. Revista Brasileira de Fruticultura, Cruz das Almas, v. 17, n. 2, p. 141-142, 1995.
ABRAHÃO, E.; ALVARENGA, A. A.; SOUZA, M. de. Comparação entre doces produzidos a partir dos frutos de marmeleiros 'Japonês' e 'Portugal'. Revista Brasileira de Fruticultura, Cruz das Almas, v. 18, n. 3, p. 441-443, 1996a.

ABRAHÃO, E.; SOUZA, M. de; ALVARENGA, A. A. A cultura do marmeleiro em Minas Gerais. Belo Horizonte: Epamig, 1996b. 23 p. (Boletim técnico, 47).

AGRIANUAL. Anuário estatístico da agricultura. São Paulo: FNP Consultoria \& Agroinformativos, 2004. 496 p.

ALVARENGA, A. A.; ABRAHÃO, E.; SOUZA, M. de; CARVALHO, V. D. de; LOPES, P. S. N.; GONÇALVES, C. A. A. Caracterização físico-química dos frutos do marmeleiro Japonês (Chaenomeles sinensis Koehne.). Revista Ciência e Prática, Lavras, v. 18, n. 2, p. 178-180, 1994.

FERREIRA, M. P. L. V. O.; PESTANA, N.; ALVE, M. R.; MOTA, F. J. M.; REU, C.; CUNHA, S.; OLIVEIRA, M. B. P. P. Quince jam quality: microbiological, physicochemical and sensory evaluation. Food Control, [S.1.], v. 15, n. 4, p. 291-295, 2004. Disponível em: <http:// www.sciencedirect.com/science>. Acesso em: 27 abr. 2005.

LINKEVICH, O. A.; MARKH, A. T. Antimicrobial proprieties of canned quince juice and puree. Disponível em: 〈http://200.179.60.195:8590/capes〉. Acesso em: $27 \mathrm{dez}$. 2005 .

MORAES, M. A. C. Métodos para avaliação sensorial dos alimentos. 5. ed. Campinas: Unicamp, 1985. 85 p.

PINHEIRO, A. C. M. Qualidade pós-colheita de banana 'Maçã' submetida ao 1-MCP. 2003. 60 p. Dissertação (Mestrado em Ciência dos Alimentos) - Universidade Federal de Lavras, Lavras, 2003.

RIGITANO, O. O marmelo e a sua cultura. São Paulo: Melhoramentos, 1957. 31 p. (ABC do Lavrador Prático, $67)$.

SILVA, B. M.; ANDRADE, P. B.; GONÇALVES, A. C.; SEBRA, R. M.; OLIVEIRA, M. B.; FERREIRA, M. A. Influence of jam processing upon contents of phenolics, organic acids and free amino acids in quince fruit (Cydonia oblonga Miller). European Food Research and Technology, [S.1.], v. 218, n. 4, p. 385-389, 2004a. Disponível em: <http:/ /200.179.60.195:8590/capes>. Acesso em: 27 dez. 2005. 
SILVA, B. M.; ANDRADE, P. B.; VAlENTAO, P.; Agricultural and Food Chemistry, Easton, v. 52, n. FERRERES, F.; SEBRA, R. M.; FERREIRA, M. A. 15, p. 4705-4712, 2004b. Disponível em: <http:// Quince (Cydonia oblonga Miller) fruit (pulp, peel, 200.179.60.195:8590/capes>. Acesso em: 27 dez. and seed) and jam: antioxidant activity. Journal of 2005. 